\title{
Almost periodic solutions for a delayed Nicholson's blowflies system with nonlinear density-dependent mortality terms and patch structure
}

Wei Chen ${ }^{1}$ and Wentao Wang ${ }^{2 *}$

\author{
"Correspondence: \\ wwt@mail.zjxu.edu.cn \\ ${ }^{2}$ College of Mathematics, Physics \\ and Information Engineering, \\ Jiaxing University, Jiaxing, Zhejiang \\ 314001, People's Republic of China \\ Full list of author information is \\ available at the end of the article
}

\begin{abstract}
We study positive almost periodic solutions for a delayed Nicholson's blowflies system with nonlinear density-dependent mortality terms and patch structure. By applying the differential inequality technique and the Lyapunov functional, we derive sufficient conditions for the existence and global exponential stability of positive almost periodic solutions. We also give an example and its numerical simulations to support the theoretical effectiveness.
\end{abstract}

Keywords: nonlinear density-dependent mortality term; delayed Nicholson's blowflies system; patch structure; positive almost periodic solution

\section{Introduction}

To describe the population of Australian sheep-blowfly and agree well with the experimental data of Nicholson [1], Gurney et al. [2] proposed the following famous Nicholson's blowflies equation

$$
N^{\prime}(t)=-\delta N(t)+p N(t-\tau) e^{-a N(t-\tau)}
$$

Here, $N(t)$ is the size of the population at time $t, p$ is the maximum per capita daily egg production, $\frac{1}{a}$ is the size at which the population reproduces at its maximum rate, $\delta$ is the per capita daily adult death rate, and $\tau$ is the generation time. The results on the dynamics behavior of this equation and its modifications are abundant [3-21] and systematically collected and compared by Berezansky et al. [22]. In the real world phenomena, since the almost periodic variation of the environment plays a crucial role in many biological and ecological dynamical systems and is more frequent and general than the periodic variation of the environment, some influential theory and results have been obtained in [23, 24]. Furthermore, there have been extensive results on the problem of the existence of positive almost periodic solutions for Nicholson's blowflies equation without nonlinear density-dependent mortality term in the literature [25-27] which are considered only with a linear density-dependent mortality term. Recently, considering that new fishery models with nonlinear density-dependent mortality rates are successfully applied, Berezansky 
et al. [22] presented the following Nicholson's blowflies model with a nonlinear densitydependent mortality term:

$$
N^{\prime}(t)=-D(N(t))+P N(t-\tau) e^{-N(t-\tau)},
$$

where $P$ is a positive constant and the function $D$ might have one of the following forms: $D(N)=\frac{a N}{N+b}$ or $D(N)=a-b e^{-N}$ with positive constants $a, b$. Up to present, several authors in [28-35] have researched the permanence and existence of positive periodic and almost periodic solutions for model (1.2) and its generalized model. Moreover, Wang [36] studied the exponential extinction for the following delayed Nicholson's blowflies system with nonlinear density-dependent mortality terms and patch structure:

$$
N_{i}^{\prime}(t)=-D_{i i}\left(t, N_{i}(t)\right)+\sum_{j=1, j \neq i}^{n} D_{i j}\left(t, N_{j}(t)\right)+\sum_{j=1}^{l} c_{i j}(t) N_{i}\left(t-\tau_{i j}(t)\right) e^{-\gamma(i j}(t) N_{i}\left(t-\tau_{i j}(t)\right),
$$

where

$$
D_{i j}(t, N)=\frac{a_{i j}(t) N}{b_{i j}(t)+N} \quad \text { or } \quad D_{i j}(t, N)=a_{i j}(t)-b_{i j}(t) e^{-N} .
$$

However, as far as we know, there exist few works on the global exponent stability of positive almost periodic solutions for a Nicholson's blowflies system with nonlinear densitydependent mortality terms and patch structure. Motivated by the above arguments, in this paper, we investigate the existence and global exponential stability of positive almost periodic solutions for the following delayed Nicholson's blowflies system with nonlinear density-dependent mortality terms and patch structure:

$$
\begin{aligned}
N_{i}^{\prime}(t)= & -a_{i i}(t)+b_{i i}(t) e^{-N_{i}(t)}+\sum_{j=1, j \neq i}^{n}\left(a_{i j}(t)-b_{i j}(t) e^{-N_{j}(t)}\right) \\
& +\sum_{j=1}^{l} c_{i j}(t) N_{i}\left(t-\tau_{i j}(t)\right) e^{-\gamma_{i j}(t) N_{i}\left(t-\tau_{i j}(t)\right)},
\end{aligned}
$$

where $a_{i j}, b_{i j}, c_{i m}, \gamma_{i m}: R \rightarrow(0,+\infty)$ and $\tau_{i m}: R \rightarrow R_{+}$are continuous almost periodic functions with $i, j \in \Lambda:=\{1,2, \ldots, n\}, m \in \Upsilon:=\{1,2, \ldots, l\}$. It is easy to see that (1.4) is a special case of (1.3) with $D(N)=a-b e^{-N}$.

It is convenient and simple to introduce some notations. Given a bounded continuous function $f$ defined on $R$, we denote $f^{+}$and $f^{-}$as

$$
f^{-}=\inf _{t \in R} f(t), \quad f^{+}=\sup _{t \in R} f(t) .
$$

It will be assumed that $r_{i}=\max _{1 \leq j \leq l} \tau_{i j}^{+}>0$ and, without loss of generality (after scaling), that $\gamma_{i j}^{-} \geq 1, i \in \Lambda, j \in \Upsilon$. Let $R^{n}\left(R_{+}^{n}\right)$ be the set of all (nonnegative) real vectors, we will use $x=\left(x_{1}, \ldots, x_{n}\right)^{T} \in R^{n}$ to denote a column vector, in which the symbol $\left({ }^{T}\right)$ denotes the transpose of a vector. We let $|x|$ denote the absolute-value vector given by $|x|=\left(\left|x_{1}\right|, \ldots,\left|x_{n}\right|\right)^{T}$ and define $\|x\|=\max _{1 \leq i \leq n}\left|x_{i}\right|$. Denote $C=\prod_{i=1}^{n} C\left(\left[-r_{i}, 0\right], R\right)$ and $C_{+}=\prod_{i=1}^{n} C\left(\left[-r_{i}, 0\right], R_{+}\right)$as a Banach space equipped with the supremum norm defined 
by $\|\varphi\|=\sup _{-r_{i} \leq t \leq 0} \max _{1 \leq i \leq n}\left|\varphi_{i}(t)\right|$ for all $\varphi(t)=\left(\varphi_{1}(t), \ldots, \varphi_{n}(t)\right)^{T} \in C$ (or $\left.\in C_{+}\right)$. If $x_{i}(t)$ is defined on $\left[t_{0}-r_{i}, v\right)$ with $t_{0}, v \in R$ and $i \in \Lambda$, then we define $x_{t} \in C$ as $x_{t}=\left(x_{t}^{1}, \ldots, x_{t}^{n}\right)^{T}$, where $x_{t}^{i}(\theta)=x_{i}(t+\theta)$ for all $\theta \in\left[-r_{i}, 0\right]$ and $i \in \Lambda$.

It is biologically reasonable to assume that only positive solutions of model (1.4) are meaningful and therefore admissible. So we consider the admissible initial conditions

$$
N_{t_{0}}=\varphi, \quad \varphi=\left(\varphi_{1}, \ldots, \varphi_{n}\right)^{T} \in C_{+} \text {and } \varphi_{i}(0)>0, i \in \Lambda
$$

Define a continuous map $f=\left(f_{1}, f_{2}, \ldots, f_{n}\right)^{T}: R \times C_{+} \rightarrow R$ by setting

$$
\begin{aligned}
f_{i}(t, \varphi)= & -a_{i i}(t)+b_{i i}(t) e^{-\varphi_{i}(0)}+\sum_{j=1, j \neq i}^{n}\left(a_{i j}(t)-b_{i j}(t) e^{-\varphi_{j}(0)}\right) \\
& +\sum_{j=1}^{l} c_{i j}(t) \varphi_{i}\left(-\tau_{i j}(t)\right) e^{-\gamma_{i j}(t) \varphi_{i}\left(-\tau_{i j}(t)\right)}, \quad i \in \Lambda .
\end{aligned}
$$

Then $f$ is a locally Lipschitz map with respect to $\varphi \in C_{+}$, which ensures the existence and uniqueness of the solution of (1.4) with admissible initial conditions (1.5).

We denote $N_{t}\left(t_{0}, \varphi\right)\left(N\left(t ; t_{0}, \varphi\right)\right)$ for a solution of the initial value problem (1.4) and (1.5). Also, let $\left[t_{0}, \eta(\varphi)\right)$ be the maximal right-interval of existence of $N_{t}\left(t_{0}, \varphi\right)$.

The remaining part of this paper is structured as follows. We devote Section 2 to some definitions and lemmas on the bounded set and almost periodicity for system (1.4) which help to deduce the existence, uniqueness and global exponential stability of positive almost periodic solutions in Section 3. In Section 4 an example and its numerical simulations are provided to verify our results obtained in the previous sections.

\section{Preliminary results}

As it is easy to analyze the property of functions $\frac{1-x}{e^{x}}$ and $x e^{-x}$ in the range $R_{+}$, one can get that there exist only $\kappa \in(0,1)$ and $\tilde{\kappa} \in(1,+\infty)$ such that

$$
\frac{1-\kappa}{e^{\kappa}}=\frac{1}{e^{2}}, \quad \sup _{x \geq \kappa}\left|\frac{1-x}{e^{x}}\right|=\frac{1}{e^{2}}, \quad \kappa e^{-\kappa}=\widetilde{\kappa} e^{-\widetilde{\kappa}} .
$$

The following definitions and lemmas will be used to prove our main results in Section 3.

Definition 2.1 (see $[23,24]$ ) Let $u(t): R^{1} \longrightarrow R^{n}$ be continuous in $t . u(t)$ is said to be almost periodic on $R^{1}$ if for any $\varepsilon>0$, the set $T(u, \varepsilon)=\left\{\delta:\|u(t+\delta)-u(t)\|<\varepsilon\right.$ for all $\left.t \in R^{1}\right\}$ is relatively dense, i.e., for any $\varepsilon>0$, it is possible to find a real number $l=l(\varepsilon)>0$ such that for any interval with length $l(\varepsilon)$, there exists a number $\delta=\delta(\varepsilon)$ in this interval such that $\|u(t+\delta)-u(t)\|<\varepsilon$ for all $t \in R^{1}$. From the theory of almost periodic functions in [23, 24], it follows that for any $\varepsilon>0$, it is possible to find a real number $l=l(\varepsilon)>0$; for any interval with length $l(\varepsilon)$, there exists a number $\delta=\delta(\varepsilon)$ in this interval such that

$$
\left\{\begin{array}{l}
\left|a_{i j}(t+\delta)-a_{i j}(t)\right|<\varepsilon, \quad\left|b_{i j}(t+\delta)-b_{i j}(t)\right|<\varepsilon, \\
\left|c_{i m}(t+\delta)-c_{i m}(t)\right|<\varepsilon, \\
\left|\tau_{i m}(t+\delta)-\tau_{i m}(t)\right|<\varepsilon, \quad\left|\gamma_{i m}(t+\delta)-\gamma_{i m}(t)\right|<\varepsilon
\end{array}\right.
$$

for all $t \in R, i, j \in \Lambda$ and $m \in \Upsilon$. 
Lemma 2.1 Suppose that there exists a positive constant $M$ such that

$$
\left.\begin{array}{l}
\gamma_{i j}^{+} \leq \frac{\tilde{\kappa}}{M}, \quad i \in \Lambda, j \in \Upsilon, \\
\sup _{t \in R}\left\{-a_{i i}(t)+b_{i i}(t) e^{-M}+\sum_{j=1, j \neq i}^{n} a_{i j}(t)+\sum_{j=1}^{l} \frac{c_{i j}(t)}{\gamma_{i j}(t)} \frac{1}{e}\right\}<0, \quad i \in \Lambda, \\
\inf _{t \in R, s \in[0, \kappa]}\left\{-a_{i i}(t)+b_{i i}(t) e^{-s}+\sum_{j=1, j \neq i}^{n}\left(a_{i j}(t)-b_{i j}(t)\right)\right. \\
\left.\quad+\sum_{j=1}^{l} \frac{c_{i j}(t)}{\gamma_{i j}(t)} s e^{-s}\right\}>0, \quad i \in \Lambda .
\end{array}\right\}
$$

Then every solution $N\left(t ; t_{0}, \varphi\right)$ of $(1.4)$ and $(1.5)$ is positive and bounded on $\left[t_{0}, \eta(\varphi)\right)$ and $\eta(\varphi)=+\infty$. Moreover, there exists $t_{\varphi}>t_{0}$ such that

$$
\kappa<N_{i}\left(t ; t_{0}, \varphi\right)<M \quad \text { for all } t \geq t_{\varphi}, i \in \Lambda .
$$

Proof Let $N(t)=N\left(t ; t_{0}, \varphi\right)=\left(N_{1}(t), N_{2}(t), \ldots, N_{n}(t)\right)^{T}$ for all $t \in\left[t_{0}, \eta(\varphi)\right)$. Firstly, we assert that

$$
N_{i}(t)>0 \quad \text { for all } t \in\left[t_{0}, \eta(\varphi)\right), i \in \Lambda \text {. }
$$

With the reduction to absurdity, assume that there exist $s_{1} \in\left[t_{0}, \eta(\varphi)\right)$ and $i \in \Lambda$ such that

$$
N_{i}\left(s_{1}\right)=0, \quad N_{j}(t)>0 \quad \text { for all } t \in\left[t_{0}, s_{1}\right), j \in \Lambda .
$$

Calculating the derivative of $N_{i}(t)$, (1.4), the second inequalities of (2.4) and (2.7) imply that

$$
\begin{aligned}
0 \geq & N_{i}^{\prime}\left(s_{1}\right) \\
= & -a_{i i}\left(s_{1}\right)+b_{i i}\left(s_{1}\right) e^{-N_{i}\left(s_{1}\right)}+\sum_{j=1, j \neq i}^{n}\left(a_{i j}\left(s_{1}\right)-b_{i j}\left(s_{1}\right) e^{-N_{j}\left(s_{1}\right)}\right) \\
& +\sum_{j=1}^{l} c_{i j}\left(s_{1}\right) N_{i}\left(s_{1}-\tau_{i j}\left(s_{1}\right)\right) e^{-\gamma_{i j}\left(s_{1}\right) N_{i}\left(s_{1}-\tau_{i j}\left(s_{1}\right)\right)} \\
\geq & -a_{i i}\left(s_{1}\right)+b_{i i}\left(s_{1}\right)+\sum_{j=1, j \neq i}^{n}\left(a_{i j}\left(s_{1}\right)-b_{i j}\left(s_{1}\right)\right) \\
\geq & \inf _{t \in R, s \in[0, \kappa]}\left\{-a_{i i}(t)+b_{i i}(t) e^{-s}+\sum_{j=1, j \neq i}^{n}\left(a_{i j}(t)-b_{i j}(t)\right)+\sum_{j=1}^{l} \frac{c_{i j}(t)}{\gamma_{i j}(t)} s e^{-s}\right\} \\
> & 0,
\end{aligned}
$$

which is paradoxical and implies that (2.6) holds.

Next we show that $N(t)$ is bounded on $\left[t_{0}, \eta(\varphi)\right)$. For each $t \in\left[t_{0}-r_{i}, \eta(\varphi)\right), i \in \Lambda$, we define

$$
M_{i}(t)=\max \left\{\xi: \xi \leq t, N_{i}(\xi)=\max _{t_{0}-r_{i} \leq s \leq t} N_{i}(s)\right\} .
$$


In the contrary case, suppose that there exists $i \in \Lambda$ such that $N_{i}(t)$ is unbounded on $\left[t_{0}, \eta(\varphi)\right)$. Then, observe $M_{i}(t) \rightarrow \eta(\varphi)$ as $t \rightarrow \eta(\varphi)$, we get

$$
\lim _{t \rightarrow \eta(\varphi)} N_{i}(M(t))=+\infty
$$

On the other hand,

$$
N_{i}(M(t))=\max _{t_{0}-r_{i} \leq s \leq t} N_{i}(s), \quad \text { and so } \quad N_{i}^{\prime}(M(t)) \geq 0, \quad \text { where } M(t)>t_{0} .
$$

Hence, together with the reality that $\sup _{u \geq 0} u e^{-u}=\frac{1}{e}$ and (1.4), we have

$$
\begin{aligned}
0 \leq & N_{i}^{\prime}(M(t)) \\
= & -a_{i i}(M(t))+b_{i i}(M(t)) e^{-N_{i}(M(t))} \\
& +\sum_{j=1, j \neq i}^{n}\left(a_{i j}(M(t))-b_{i j}(M(t)) e^{-N_{j}(M(t))}\right) \\
& +\sum_{j=1}^{l} \frac{c_{i j}(M(t))}{\gamma_{i j}(M(t))} \gamma_{i j}(M(t)) N_{i}\left(M(t)-\tau_{i j}(M(t))\right) e^{-\gamma_{i j}(M(t)) N_{i}\left(M(t)-\tau_{i j}(M(t))\right)} \\
\leq & -a_{i i}(M(t))+b_{i i}(M(t)) e^{-N_{i}(M(t))}+\sum_{j=1, j \neq i}^{n} a_{i j}(M(t)) \\
& +\sum_{j=1}^{l} \frac{c_{i j}(M(t))}{\gamma_{i j}(M(t))} \frac{1}{e}, \quad \text { where } M(t)>t_{0} .
\end{aligned}
$$

Taking $t \rightarrow \eta(\varphi)$ results in

$$
\begin{aligned}
0 & \leq \lim _{t \rightarrow \eta(\varphi)}\left[-a_{i i}(t)+\sum_{j=1, j \neq i}^{n} a_{i j}(t)+\sum_{j=1}^{l} \frac{c_{i j}(t)}{\gamma_{i j}(t)} \frac{1}{e}\right] \\
& \leq \sup _{t \in R}\left\{-a_{i i}(t)+b_{i i}(t) e^{-M}+\sum_{j=1, j \neq i}^{n} a_{i j}(t)+\sum_{j=1}^{l} \frac{c_{i j}(t)}{\gamma_{i j}(t)} \frac{1}{e}\right\} \\
& <0,
\end{aligned}
$$

which is absurd and implies that $N(t)$ is bounded on $\left[t_{0}, \eta(\varphi)\right)$. From Theorem 2.3.1 in [37], we easily obtain $\eta(\varphi)=+\infty$.

Another step is to prove that there exists $s_{2} \in\left[t_{0},+\infty\right)$ such that

$$
N_{i}\left(s_{2}\right)<M, \quad i \in \Lambda \text {. }
$$

Otherwise, there exists $i \in \Lambda$ such that

$$
N_{i}(t) \geq M \quad \text { for all } t \in\left[t_{0},+\infty\right),
$$


which together with the first inequalities of (2.4) and (2.6) implies that

$$
\begin{aligned}
N_{i}^{\prime}(t)= & -a_{i i}(t)+b_{i i}(t) e^{-N_{i}(t)}+\sum_{j=1, j \neq i}^{n}\left(a_{i j}(t)-b_{i j}(t) e^{-N_{j}(t)}\right) \\
& +\sum_{j=1}^{l} \frac{c_{i j}(t)}{\gamma_{i j}(t)} \gamma_{i j}(t) N_{i}\left(t-\tau_{i j}(t)\right) e^{-\gamma_{i j}(t) N_{i}\left(t-\tau_{i j}(t)\right)} \\
\leq & -a_{i i}(t)+b_{i i}(t) e^{-M}+\sum_{j=1, j \neq i}^{n} a_{i j}(t)+\sum_{j=1}^{l} \frac{c_{i j}(t)}{\gamma_{i j}(t)} \frac{1}{e} \\
< & 0 \quad \text { for all } t \geq t_{0} .
\end{aligned}
$$

It leads to

$$
\begin{aligned}
N_{i}(t)= & N_{i}\left(t_{0}\right)+\int_{t_{0}}^{t} N_{i}^{\prime}(s) d s \\
\leq & N_{i}\left(t_{0}\right)+\sup _{t \in R}\left\{-a_{i i}(t)+b_{i i}(t) e^{-M}+\sum_{j=1, j \neq i}^{n} a_{i j}(t)+\sum_{j=1}^{l} \frac{c_{i j}(t)}{\gamma_{i j}(t)} \frac{1}{e}\right\} \\
& \times\left(t-t_{0}\right) \quad \text { for all } t \geq t_{0} .
\end{aligned}
$$

Thus

$$
\lim _{t \rightarrow+\infty} N_{i}(t)=-\infty
$$

which contradicts (2.6). Hence (2.9) holds. We now prove that

$$
N_{i}(t)<M \quad \text { for all } t \in\left[s_{2},+\infty\right), i \in \Lambda \text {. }
$$

Suppose, for the sake of contradiction, that there exist $s_{3} \in\left(s_{2},+\infty\right)$ and $i \in \Lambda$ such that

$$
N_{i}\left(s_{3}\right)=M, \quad N_{j}(t)<M \quad \text { for all } t \in\left[s_{2}, s_{3}\right), j \in \Lambda .
$$

Calculating the derivative of $N_{i}(t)$, together with the fact that $\sup _{x \in R} x e^{-x}=\frac{1}{e},(1.4)$, the first inequalities of (2.4) and (2.11) imply that

$$
\begin{aligned}
0 \leq & N_{i}^{\prime}\left(s_{3}\right) \\
= & -a_{i i}\left(s_{3}\right)+b_{i i}\left(s_{3}\right) e^{-N_{i}\left(s_{3}\right)}+\sum_{j=1, j \neq i}^{n}\left(a_{i j}\left(s_{3}\right)-b_{i j}\left(s_{3}\right) e^{-N_{j}\left(s_{3}\right)}\right) \\
& +\sum_{j=1}^{l} \frac{c_{i j}\left(s_{3}\right)}{\gamma_{i j}\left(s_{3}\right)} \gamma_{i j}\left(s_{3}\right) N_{i}\left(s_{3}-\tau_{i j}\left(s_{3}\right)\right) e^{-\gamma_{i j}\left(s_{3}\right) N_{i}\left(s_{3}-\tau_{i j}\left(s_{3}\right)\right)} \\
\leq & -a_{i i}\left(s_{3}\right)+b_{i i}\left(s_{3}\right) e^{-M}+\sum_{j=1, j \neq i}^{n} a_{i j}\left(s_{3}\right)+\sum_{j=1}^{l} \frac{c_{i j}\left(s_{3}\right)}{\gamma_{i j}\left(s_{3}\right)} \frac{1}{e} \\
< & 0,
\end{aligned}
$$

which is a contradiction and implies that (2.10) holds. 
Finally, we show that $l_{i}=\liminf _{t \rightarrow+\infty} N_{i}(t)>\kappa$ for all $i \in \Lambda$. By a way of contradiction, we assume that there exists $i \in \Lambda$ such that $0 \leq l_{i} \leq \kappa$. By the fluctuation lemma [38, Lemma A.1.], there exists a sequence $\left\{t_{k}\right\}_{k \geq 1}$ such that

$$
t_{k} \rightarrow+\infty, \quad N_{i}\left(t_{k}\right) \rightarrow \liminf _{t \rightarrow+\infty} N_{i}(t), \quad N_{i}^{\prime}\left(t_{k}\right) \rightarrow 0 \quad \text { as } k \rightarrow+\infty
$$

Since $\left\{N_{t_{k}}\right\}_{k \geq 1}$ is bounded and equicontinuous, by the Ascoli-Arzelà theorem, there exists a subsequence, still denoted by itself for simplicity of notation, such that

$$
N_{t_{k}} \rightarrow \varphi^{*} \quad \text { for some } \varphi^{*} \in C_{+} \text {. }
$$

Moreover,

$$
\varphi_{j}^{*}(0)=l_{j} \leq \varphi_{j}^{*}(\theta) \leq M \quad \text { for } \theta \in\left[-r_{j}, 0\right), j \in \Lambda
$$

Without loss of generality, we assume that all $a_{i j}\left(t_{k}\right), b_{i j}\left(t_{k}\right), c_{i m}\left(t_{k}\right), \gamma_{i m}\left(t_{k}\right)$ and $\tau_{i m}\left(t_{k}\right)$ are convergent to $a_{i j}^{*}, b_{i j}^{*}, c_{i m}^{*}, \gamma_{i m}^{*}$ and $\tau_{i m}^{*}$, respectively, and $i, j \in \Lambda, m \in \Upsilon$. This can be achieved because of almost periodicity. Then by (1.5) and (2.3) we arrive at

$$
l_{i} \leq \gamma_{i j}^{*} \varphi_{i}^{*}\left(-\tau_{i j}^{*}\right) \leq \gamma_{i j}^{*} M \leq \widetilde{\kappa}, \quad j \in \Upsilon .
$$

It follows from

$$
\begin{aligned}
N_{i}^{\prime}\left(t_{k}\right)= & -a_{i i}\left(t_{k}\right)+b_{i i}\left(t_{k}\right) e^{-N_{i}\left(t_{k}\right)}+\sum_{j=1, j \neq i}^{n}\left(a_{i j}\left(t_{k}\right)-b_{i j}\left(t_{k}\right) e^{-N_{j}\left(t_{k}\right)}\right) \\
& +\sum_{j=1}^{l} \frac{c_{i j}\left(t_{k}\right)}{\gamma_{i j}\left(t_{k}\right)} \gamma_{i j}\left(t_{k}\right) N_{i}\left(t_{k}-\tau_{i j}\left(t_{k}\right)\right) e^{-\gamma_{i j}\left(t_{k}\right) N_{i}\left(t_{k}-\tau_{i j}\left(t_{k}\right)\right)}
\end{aligned}
$$

that (taking limits)

$$
\begin{aligned}
0 & =-a_{i i}^{*}+b_{i i}^{*} e^{-l_{i}}+\sum_{j=1, j \neq i}^{n}\left(a_{i j}^{*}-b_{i j}^{*} e^{-\varphi_{j}^{*}(0)}\right)+\sum_{j=1}^{l} \frac{c_{i j}^{*}}{\gamma_{i j}^{*}} \gamma_{i j}^{*} \varphi_{i}^{*}\left(-\tau_{i j}^{*}\right) e^{-\gamma_{i j}^{*} \varphi_{i}^{*}\left(-\tau_{i j}^{*}\right)} \\
& \geq-a_{i i}^{*}+b_{i i}^{*} e^{-l_{i}}+\sum_{j=1, j \neq i}^{n}\left(a_{i j}^{*}-b_{i j}^{*}\right)+\sum_{j=1}^{l} \frac{c_{i j}^{*}}{\gamma_{i j}^{*}} l_{i} e^{-l_{i}} \\
& \geq \inf _{t \in R, s \in[0, \kappa]}\left\{-a_{i i}(t)+b_{i i}(t) e^{-s}+\sum_{j=1, j \neq i}^{n}\left(a_{i j}(t)-b_{i j}(t)\right)+\sum_{j=1}^{l} \frac{c_{i j}(t)}{\gamma_{i j}(t)} s e^{-s}\right\} \\
& >0,
\end{aligned}
$$

which is a contradiction. This proves that $l_{i}>\kappa$ for all $i \in \Lambda$. Hence, from (2.10), we can choose $t_{\varphi}>t_{0}$ such that

$$
\kappa<N_{i}\left(t ; t_{0}, \varphi\right)<M \quad \text { for all } t \geq t_{\varphi}, i \in \Lambda \text {. }
$$

The proof is now completed. 
Lemma 2.2 Suppose that (2.3) and (2.4) hold, and

$$
\sup _{t \in R}\left\{-b_{i i}(t) e^{-M}+\sum_{j=1, j \neq i}^{n} b_{i j}(t) e^{-\kappa}+\frac{1}{e^{2}} \sum_{j=1}^{l} c_{i j}(t)\right\}<0, \quad i \in \Lambda \text {. }
$$

Moreover, let $N(t)=N\left(t ; t_{0}, \varphi\right)=\left(N_{1}(t), N_{2}(t), \ldots, N_{n}(t)\right)^{T}$ be a solution of equation (1.4) with initial condition (1.5) and $\varphi_{i}^{\prime}$ is bounded continuous on $\left[-r_{i}, 0\right], i \in \Lambda$. Then, for any $\varepsilon>0$, there exists $l=l(\varepsilon)>0$ such that every interval $[\alpha, \alpha+l]$ contains at least one number $\delta$ for which there exists $N>0$ satisfies

$$
\|N(t+\delta)-N(t)\| \leq \varepsilon \quad \text { for all } t>N
$$

Proof For all $i \in \Lambda$, define continuous functions $\Gamma_{i}(u)$ by setting

$$
\Gamma_{i}(u)=-\left[b_{i i}(t) e^{-M}-u\right]+\sum_{j=1, j \neq i}^{n} b_{i j}(t) e^{-\kappa}+\frac{1}{e^{2}} \sum_{j=1}^{l} c_{i j}(t) e^{u r_{i}} \quad \text { for all } t \in R, u \in[0,1]
$$

Then, from (2.12), we obtain

$$
\Gamma_{i}(0)=-b_{i i}(t) e^{-M}+\sum_{j=1, j \neq i}^{n} b_{i j}(t) e^{-\kappa}+\frac{1}{e^{2}} \sum_{j=1}^{l} c_{i j}(t)<0 \quad \text { for all } t \in R, i \in \Lambda,
$$

which implies that there exist two constants $\eta>0$ and $\lambda \in(0,1]$ such that

$$
\begin{aligned}
\Gamma_{i}(\lambda) & =-\left[b_{i i}(t) e^{-M}-\lambda\right]+\sum_{j=1, j \neq i}^{n} b_{i j}(t) e^{-\kappa}+\frac{1}{e^{2}} \sum_{j=1}^{l} c_{i j}(t) e^{\lambda r_{i}} \\
& <-\eta<0 \quad \text { for all } t \in R, i \in \Lambda .
\end{aligned}
$$

For $i \in \Lambda, t \in\left(-\infty, t_{0}-r_{i}\right]$, we add the definition of $N_{i}(t)$ with $N_{i}(t) \equiv N_{i}\left(t_{0}-r_{i}\right)$. Set

$$
\begin{aligned}
\varepsilon_{i}(\delta, t)= & {\left[b_{i i}(t+\delta)-b_{i i}(t)\right] e^{-N_{i}(t+\delta)}-\sum_{j=1, j \neq i}^{n}\left[b_{i j}(t+\delta)-b_{i j}(t)\right] e^{-N_{j}(t+\delta)} } \\
& +\sum_{j=1}^{l}\left[c_{i j}(t+\delta)-c_{i j}(t)\right] N_{i}\left(t+\delta-\tau_{i j}(t+\delta)\right) e^{-\gamma_{i j}(t+\delta) N_{i}\left(t+\delta-\tau_{i j}(t+\delta)\right)} \\
& +\sum_{j=1}^{l} c_{i j}(t)\left[N_{i}\left(t+\delta-\tau_{i j}(t+\delta)\right) e^{-\gamma_{i j}(t+\delta) N_{i}\left(t+\delta-\tau_{i j}(t+\delta)\right)}\right. \\
& \left.-N_{i}\left(t-\tau_{i j}(t)+\delta\right) e^{-\gamma_{i j}(t+\delta) N_{i}\left(t-\tau_{i j}(t)+\delta\right)}\right] \\
& +\sum_{j=1}^{l} c_{i j}(t)\left[N_{i}\left(t-\tau_{i j}(t)+\delta\right) e^{-\gamma_{i j}(t+\delta) N_{i}\left(t-\tau_{i j}(t)+\delta\right)}\right. \\
& \left.-N_{i}\left(t-\tau_{i j}(t)+\delta\right) e^{-\gamma_{i j}(t) N_{i}\left(t-\tau_{i j}(t)+\delta\right)}\right]-\left[a_{i i}(t+\delta)-a_{i i}(t)\right] \\
& +\sum_{j=1, j \neq i}^{n}\left[a_{i j}(t+\delta)-a_{i j}(t)\right], \quad t \in R .
\end{aligned}
$$


By Lemma 2.1, the solution $N(t)$ is bounded and

$$
\kappa<N_{i}(t)<M \text { for all } t \geq t_{\varphi}, i \in \Lambda,
$$

which implies that the right-hand side of (1.4) is also bounded, and $N_{i}^{\prime}(t)$ is a bounded function on $\left[t_{0}-r_{i},+\infty\right), i \in \Lambda$. Thus, in view of the fact that $N_{i}(t) \equiv N_{i}\left(t_{0}-r_{i}\right)$ for $t \in$ $\left(-\infty, t_{0}-r_{i}\right], i \in \Lambda$, we obtain that $N_{i}(t)$ is uniformly continuous on $R$. From (2.2), for any $\varepsilon$, there exists $l=l(\varepsilon)>0$ such that every interval $[\alpha, \alpha+l]$ contains $\delta$ for which

$$
\left|\varepsilon_{i}(\delta, t)\right| \leq \frac{1}{2} \eta \varepsilon \quad \text { for all } t \in R, i \in \Lambda
$$

Let $N_{0} \geq \max \left\{t_{0}, t_{0}-\delta, t_{\varphi}+\max _{i \in \Lambda} r_{i}\right\}$ and denote $u(t)=\left(u_{1}(t), u_{2}(t), \ldots, u_{n}(t)\right)^{T}$, where $u_{i}(t)=N_{i}(t+\delta)-N_{i}(t), i \in \Lambda$. Then, for all $t \geq N_{0}$, we have

$$
\begin{aligned}
u_{i}^{\prime}(t)= & b_{i i}(t)\left[e^{-N_{i}(t+\delta)}-e^{-N_{i}(t)}\right]-\sum_{j=1, j \neq i}^{n} b_{i j}(t)\left[e^{-N_{j}(t+\delta)}-e^{-N_{j}(t)}\right] \\
& +\sum_{j=1}^{l} c_{i j}(t)\left[N_{i}\left(t-\tau_{i j}(t)+\delta\right) e^{-\gamma_{i j}(t) N_{i}\left(t-\tau_{i j}(t)+\delta\right)}\right. \\
& \left.-N_{i}^{*}\left(t-\tau_{i j}(t)\right) e^{-\gamma_{i j}(t) N_{i}^{*}\left(t-\tau_{i j}(t)\right)}\right]+\varepsilon_{i}(\delta, t) .
\end{aligned}
$$

Set

$$
U(t)=\left(U_{1}(t), U_{2}(t), \ldots, U_{n}(t)\right)^{T}, \quad \text { where } U_{i}(t)=e^{\lambda t} u_{i}(t), i \in \Lambda
$$

Let $i_{t}$ be such an index that

$$
\left|U_{i_{t}}(t)\right|=\|U(t)\|
$$

Calculating the upper left derivative of $\left|U_{i_{s}}(s)\right|$ along with (2.18), together with (2.1), (2.15), (2.16), (2.19) and the inequalities

$$
\begin{aligned}
\left(e^{-s}-e^{-t}\right) \operatorname{sgn}(s-t) & =-e^{-s+\theta(s-t)}|s-t| \\
\leq & -e^{-M}|s-t|, \quad \text { where } s, t \in[\kappa, M], 0<\theta<1, \\
\left|e^{-s}-e^{-t}\right| & =e^{-s+\theta(s-t)}|s-t| \\
\leq & e^{-\kappa}|s-t|, \quad \text { where } s, t \in[\kappa,+\infty], 0<\theta<1,
\end{aligned}
$$

and

$$
\begin{aligned}
\left|s e^{-s}-t e^{-t}\right| & =\left|\frac{1-(s+\theta(t-s))}{e^{s+\theta(t-s)}}\right||s-t| \\
& \leq \frac{1}{e^{2}}|s-t|, \quad \text { where } s, t \in[\kappa,+\infty], 0<\theta<1,
\end{aligned}
$$


we get

$$
\begin{aligned}
& \left.D^{-}\left(\left|U_{i_{s}}(s)\right|\right)\right|_{s=t} \\
& \leq \lambda e^{\lambda t}\left|u_{i_{t}}(t)\right|+e^{\lambda t}\left\{b_{i_{t} i_{t}}(t)\left[e^{-N_{i_{t}}(t+\delta)}-e^{-N_{i_{t}}(t)}\right] \operatorname{sgn}\left(N_{i_{t}}(t+\delta)-N_{i_{t}}(t)\right)\right. \\
& +\sum_{j=1, j \neq i i_{t}}^{n} b_{i_{t} j}(t)\left|e^{-N_{j}(t+\delta)}-e^{-N_{j}(t)}\right|+\sum_{j=1}^{l} c_{i_{t} j}(t) \\
& \times\left|N_{i_{t}}\left(t-\tau_{i_{t} j}(t)+\delta\right) e^{-\gamma_{i_{t} j}(t) N_{i_{t}}\left(t-\tau_{i_{t} j}(t)+\delta\right)}-N_{i_{t}}\left(t-\tau_{i_{t} j}(t)\right) e^{-\gamma_{i_{t} j}(t) N_{i_{t}}\left(t-\tau_{i_{t} j}(t)\right)}\right| \\
& \left.+\left|\varepsilon_{i_{t}}(\delta, t)\right|\right\} \\
& =\lambda e^{\lambda t}\left|u_{i_{t}}(t)\right|+e^{\lambda t}\left\{b_{i_{t} i_{t}}(t)\left[e^{-N_{i_{t}}(t+\delta)}-e^{-N_{i_{t}}(t)}\right] \operatorname{sgn}\left(N_{i_{t}}(t+\delta)-N_{i_{t}}(t)\right)\right. \\
& +\sum_{j=1, j \neq i_{t}}^{n} b_{i_{t} j}(t)\left|e^{-N_{j}(t+\delta)}-e^{-N_{j}(t)}\right|+\sum_{j=1}^{l} \frac{c_{i_{t} j}(t)}{\gamma_{i_{t} j}(t)} \\
& \times \mid \gamma_{i_{t} j}(t) N_{i_{t}}\left(t-\tau_{i_{t} j}(t)+\delta\right) e^{-\gamma_{i_{t} j}(t) N_{i_{t}}\left(t-\tau_{i_{t} j}(t)+\delta\right)} \\
& \left.-\gamma_{i_{t} j}(t) N_{i_{t}}\left(t-\tau_{i_{t} j}(t)\right) e^{-\gamma_{i_{t} j}(t) N_{i_{t}}\left(t-\tau_{i_{t} j}(t)\right)}|+| \varepsilon_{i_{t}}(\delta, t) \mid\right\} \\
& \leq \lambda e^{\lambda t}\left|u_{i_{t}}(t)\right|+e^{\lambda t}\left\{-b_{i_{t} i_{t}}(t) e^{-M}\left|U_{i_{t}}(t)\right|+\sum_{j=1, j \neq i_{t}}^{n} b_{i_{t} j}(t) e^{-\kappa}\left|u_{j}(t)\right|\right. \\
& \left.+\sum_{j=1}^{l} \frac{c_{i_{t} j}(t)}{\gamma_{i_{t} j}(t)} \frac{1}{e^{2}}\left|u_{i_{t}}\left(t-\tau_{i_{t} j}(t)\right)\right|+\left|\varepsilon_{i_{t}}(\delta, t)\right|\right\} \\
& =-\left[b_{i_{t} i_{t}}(t) e^{-M}-\lambda\right]\left|U_{i_{t}}(t)\right|+\sum_{j=1, j \neq i_{t}}^{n} b_{i_{t} j}(t) e^{-\kappa}\left|U_{j}(t)\right| \\
& +\sum_{j=1}^{l} \frac{c_{i_{t} j}(t)}{\gamma_{i_{t} j}(t)} \frac{1}{e^{2}} e^{\lambda \tau_{i_{t}}(t)}\left|U_{i_{t}}\left(t-\tau_{i_{t} j}(t)\right)\right|+e^{\lambda t}\left|\varepsilon_{i_{t}}(\delta, t)\right| \quad \text { for all } t \geq N_{0}
\end{aligned}
$$

which is held under the following fact:

$$
\kappa \leq \gamma_{i_{t} j}(t) N_{i_{t}}\left(t-\tau_{i_{t} j}(t)+\delta\right), \gamma_{i_{t}}(t) N_{i_{t}}^{*}\left(t-\tau_{i j}(t)\right) \leq \gamma_{i_{t j}}^{+} M \leq \widetilde{\kappa}, \quad j=1,2, \ldots, l, t \geq N_{0} .
$$

Let

$$
M(t)=\max _{s \leq t}\{\|U(s)\|\}
$$

It is obvious that $M(t) \geq\|U(t)\|$ and $M(t)$ is non-decreasing. Now, we distinguish two cases to finish the proof.

Case one.

$$
M(t)>\|U(t)\| \quad \text { for all } t \geq N_{0}
$$


We claim that

$$
M(t) \equiv M\left(N_{0}\right) \quad \text { is a constant for all } t \geq N_{0} .
$$

Assume, by a way of contradiction, that (2.26) does not hold. Then there exists $s_{4}>N_{0}$ such that $M\left(s_{4}\right)>M\left(N_{0}\right)$. Since

$$
M\left(N_{0}\right) \geq\|U(t)\| \quad \text { for all } t \leq N_{0},
$$

there must exist $\beta \in\left(N_{0}, s_{4}\right)$ such that

$$
\|U(\beta)\|=M\left(s_{4}\right) \geq M(\beta),
$$

which contradicts (2.25) and implies that (2.26) holds. It follows that there exists $s_{5}>N_{0}$ such that

$$
\|u(t)\| \leq e^{-\lambda t} M(t)=e^{-\lambda t} M\left(N_{0}\right)<\varepsilon \quad \text { for all } t \geq s_{5} .
$$

Case two. There is $\rho>N_{0}$ such that $M(\rho)=\|U(\rho)\|$. Then, in view of (2.14), (2.17) and (2.23), we have

$$
\begin{aligned}
0 \leq & \left.D^{-}\left(\left|U_{i_{s}}(s)\right|\right)\right|_{s=\rho} \\
\leq & -\left[b_{i_{\rho} i_{\rho}}(\rho) e^{-M}-\lambda\right]\left|U_{i_{\rho}}(\rho)\right|+\sum_{j=1, j \neq i_{\rho}}^{n} b_{i_{\rho} j}(\rho) e^{-\kappa}\left|U_{j}(\rho)\right| \\
& +\sum_{j=1}^{l} \frac{c_{i_{\rho} j}(\rho)}{\gamma_{i_{\rho} j}(\rho)} \frac{1}{e^{2}} e^{\lambda \tau_{i_{\rho} j}(\rho)}\left|U_{i_{\rho}}\left(\rho-\tau_{i_{\rho} j}(\rho)\right)\right|+e^{\lambda \rho}\left|\varepsilon_{i_{\rho}}(\delta, \rho)\right| \\
\leq & \left\{-\left[b_{i_{\rho} i_{\rho}}(\rho) e^{-M}-\lambda\right]+\sum_{j=1, j \neq i_{\rho}}^{n} b_{i_{\rho} j}(\rho) e^{-\kappa}\right. \\
& \left.+\sum_{j=1}^{l} \frac{1}{e^{2}} c_{i_{\rho} j}(\rho) e^{\lambda r_{i}}\right\}\|U(\rho)\|+\frac{1}{2} \eta \varepsilon e^{\lambda \rho} \\
< & -\eta\|U(\rho)\|+\eta \varepsilon e^{\lambda \rho},
\end{aligned}
$$

which yields that

$$
e^{\lambda \rho}\|u(\rho)\|=\|U(\rho)\|<\varepsilon e^{\lambda \rho} \quad \text { and } \quad\|u(\rho)\|<\varepsilon .
$$

For any $t>\rho$, with the same approach as that in deriving of (2.29), we can show

$$
e^{\lambda t}\|u(t)\|=\|U(t)\|<\varepsilon e^{\lambda t} \quad \text { and } \quad\|u(t)\|<\varepsilon
$$

if $M(t)=\|U(t)\|$. On the other hand, if $M(t)>\|U(t)\|$ and $t>\rho$, we can choose $\rho \leq s_{6}<t$ such that

$$
M\left(s_{6}\right)=\left\|U\left(s_{6}\right)\right\|, \quad \text { and } \quad M(s)>\|U(s)\| \quad \text { for all } s \in\left(s_{6}, t\right],
$$


which together with (2.30) yields

$$
\left\|u\left(s_{6}\right)\right\|<\varepsilon
$$

With a similar argument as that in the proof of case one, we can show that

$$
M(s) \equiv M\left(s_{6}\right) \quad \text { is a constant for all } s \in\left(s_{6}, t\right],
$$

which implies that

$$
\|u(t)\|<e^{-\lambda t} M(t)=e^{-\lambda t} M\left(s_{6}\right)=\left\|u\left(s_{6}\right)\right\| e^{-\lambda\left(t-s_{6}\right)}<\varepsilon .
$$

In summary, there must exist $N>\max \left\{\rho, N_{0}, s_{5}\right\}$ such that $\|u(t)\| \leq \varepsilon$ holds for all $t>N$. This completes the proof.

\section{Main results}

In this section, we establish sufficient conditions for the existence and global exponential stability of positive almost periodic solutions of (1.4).

Theorem 3.1 Suppose that all conditions in Lemma 2.2 are satisfied. Then system (1.4) has at least one positive almost periodic solution $N^{*}(t)$. Moreover, $N^{*}(t)$ is globally exponentially stable, i.e., there exist constants $\lambda>0, K_{\varphi, N^{*}}>0$ and $t_{\varphi, N^{*}}$ such that

$$
\left\|N\left(t ; t_{0}, \varphi\right)-N^{*}(t)\right\|<K_{\varphi, N^{*}} e^{-\lambda t} \text { for all } t>t_{\varphi, N^{*}} .
$$

Proof Let $v(t)=v\left(t ; t_{0}, \psi\right)=\left(v_{1}(t), v_{2}(t), \ldots, v_{n}(t)\right)^{T}$ be a solution of equation (1.4) with initial conditions satisfying the assumptions in Lemma 2.2. We also add the definition of $v(t)$ with $v_{i}(t) \equiv v_{i}\left(t_{0}-r_{i}\right)$ for all $t \in\left(-\infty, t_{0}-r_{i}\right], i \in \Lambda$. Set

$$
\begin{aligned}
\varepsilon_{i, k}(t) & \\
= & {\left[b_{i i}\left(t+t_{k}\right)-b_{i i}(t)\right] e^{-v_{i}\left(t+t_{k}\right)}-\sum_{j=1, j \neq i}^{n}\left[b_{i j}\left(t+t_{k}\right)-b_{i j}(t)\right] e^{-v_{j}\left(t+t_{k}\right)} } \\
& +\sum_{j=1}^{l}\left[c_{i j}\left(t+t_{k}\right)-c_{i j}(t)\right] v_{i}\left(t+t_{k}-\tau_{i j}\left(t+t_{k}\right)\right) e^{-\gamma_{i j}\left(t+t_{k}\right) v_{i}\left(t+t_{k}-\tau_{i j}\left(t+t_{k}\right)\right)} \\
& +\sum_{j=1}^{l} c_{i j}(t)\left[v_{i}\left(t+t_{k}-\tau_{i j}\left(t+t_{k}\right)\right) e^{-\gamma_{i j}\left(t+t_{k}\right) v_{i}\left(t+t_{k}-\tau_{i j}\left(t+t_{k}\right)\right)}\right. \\
& \left.-v_{i}\left(t-\tau_{i j}(t)+t_{k}\right) e^{-\gamma_{i j}\left(t+t_{k}\right) v_{i}\left(t-\tau_{i j}(t)+t_{k}\right)}\right] \\
& +\sum_{j=1}^{l} c_{i j}(t)\left[v_{i}\left(t-\tau_{i j}(t)+t_{k}\right) e^{-\gamma_{i j}\left(t+t_{k}\right) v_{i}\left(t-\tau_{i j}(t)+t_{k}\right)}\right. \\
& \left.-v_{i}\left(t-\tau_{i j}(t)+t_{k}\right) e^{-\gamma_{i j}(t) v_{i}\left(t-\tau_{i j}(t)+t_{k}\right)}\right]-\left[a_{i i}\left(t+t_{k}\right)-a_{i i}(t)\right] \\
& +\sum_{j=1, j \neq i}^{n}\left[a_{i j}\left(t+t_{k}\right)-a_{i j}(t)\right], \quad t \in R, i \in \Lambda,
\end{aligned}
$$


where $\left\{t_{k}\right\}$ is any sequence of real numbers. By Lemma 2.1, the solution $v(t)$ is bounded and

$$
\kappa<v_{i}(t)<M \quad \text { for all } t \geq t_{\psi}, i \in \Lambda \text {, }
$$

which implies that the right-hand side of (1.4) is also bounded, and $v_{i}^{\prime}(t)(i \in \Lambda)$ are bounded functions on $\left[t_{0}-r_{i},+\infty\right)$. Thus, in view of the fact that $v_{i}(t) \equiv v_{i}\left(t_{0}-r_{i}\right)$ for all $t \in\left(-\infty, t_{0}-r_{i}\right], i \in \Lambda$, we obtain that $v_{i}(t)(i \in \Lambda)$ are uniformly continuous on $R$. Then, from the almost periodicity of $a_{i j}, b_{i j}, c_{i m}, \gamma_{i m}$ and $\tau_{i m}$, we can select a sequence $\left\{t_{k}\right\} \rightarrow+\infty$ such that

$$
\begin{cases}\left|a_{i j}\left(t+t_{k}\right)-a_{i j}(t)\right| \leq \frac{1}{k}, & \left|b_{i j}\left(t+t_{k}\right)-b_{i j}(t)\right| \leq \frac{1}{k}, \\ \left|c_{i m}\left(t+t_{k}\right)-c_{i m}(t)\right| \leq \frac{1}{k}, & \\ \left|\tau_{i m}\left(t+t_{k}\right)-\tau_{i m}(t)\right| \leq \frac{1}{k}, & \left|\gamma_{i m}\left(t+t_{k}\right)-\gamma_{i m}(t)\right| \leq \frac{1}{k}, \quad\left|\varepsilon_{i, k}(t)\right| \leq \frac{1}{k}\end{cases}
$$

for all $t \in R$ and $i, j \in \Lambda, m \in \Upsilon$.

Since $\left\{v_{i}\left(t+t_{k}\right)\right\}_{k=1}^{+\infty}(i \in \Lambda)$ is uniformly bounded and equi-uniformly continuous, by the Arzelà-Ascoli lemma and the diagonal selection principle, we can choose a subsequence $\left\{t_{k_{j}}\right\}$ of $\left\{t_{k}\right\}$ such that $v_{i}\left(t+t_{k_{j}}\right)$ (for convenience, we still denote it by $v_{i}\left(t+t_{k}\right), i \in \Lambda$ ) uniformly converges to a continuous function $N_{i}^{*}(t)(i \in \Lambda)$ on any compact set of $R$, and

$$
\kappa \leq N_{i}^{*}(t) \leq M \quad \text { for all } t \in R, i \in \Lambda .
$$

Now, we prove that $N^{*}(t)=\left(N_{1}^{*}(t), N_{2}^{*}(t), \ldots, N_{n}^{*}(t)\right)^{T}$ is a solution of (1.4). In fact, for any $t \geq t_{0}$ and $\Delta t \in R$, from (3.3), we have

$$
\begin{aligned}
N_{i}^{*}( & t+\Delta t)-N_{i}^{*}(t) \\
= & \lim _{k \rightarrow+\infty}\left[v_{i}\left(t+\Delta t+t_{k}\right)-v_{i}\left(t+t_{k}\right)\right] \\
= & \lim _{k \rightarrow+\infty} \int_{t}^{t+\Delta t}\left[-a_{i i}\left(s+t_{k}\right)+b_{i i}\left(s+t_{k}\right) e^{-v_{i}\left(s+t_{k}\right)}+\sum_{j=1, j \neq i}^{n}\left(a_{i j}\left(s+t_{k}\right)-b_{i j}\left(s+t_{k}\right) e^{-v_{j}\left(s+t_{k}\right)}\right)\right. \\
& \left.+\sum_{j=1}^{l} c_{i j}\left(s+t_{k}\right) v_{i}\left(s+t_{k}-\tau_{i j}\left(s+t_{k}\right)\right) e^{-\gamma_{i j}\left(s+t_{k}\right) v_{i}\left(s+t_{k}-\tau_{i j}\left(s+t_{k}\right)\right)}\right] d s \\
= & \lim _{k \rightarrow+\infty} \int_{t}^{t+\Delta t}\left[-a_{i i}(s)+b_{i i}(s) e^{-v_{i}\left(s+t_{k}\right)}+\sum_{j=1, j \neq i}^{n}\left(a_{i j}(s)-b_{i j}(s) e^{-v_{j}\left(s+t_{k}\right)}\right)\right. \\
& \left.+\sum_{j=1}^{l} c_{i j}(s) v_{i}\left(s+t_{k}-\tau_{i j}(s)\right) e^{-\gamma_{i j}(s) v_{i}\left(s+t_{k}-\tau_{i j}(s)\right)}+\varepsilon_{i, k}(s)\right] d s \\
= & \int_{t}^{t+\Delta t}\left[-a_{i i}(s)+b_{i i}(s) e^{-N_{i}^{*}(s)}+\sum_{j=1, j \neq i}^{n}\left(a_{i j}(s)-b_{i j}(s) e^{-N_{j}^{*}(s)}\right)\right. \\
& \left.+\sum_{j=1}^{l} c_{i j}(s) N_{i}^{*}\left(s-\tau_{i j}(s)\right) e^{-\gamma_{i j}(s) N_{i}^{*}\left(s-\tau_{i j}(s)\right)}\right] d s,
\end{aligned}
$$


where $t+\Delta t \geq t_{0}, i \in \Lambda$. Consequently, (3.5) implies that

$$
\begin{aligned}
\frac{d}{d t}\left\{N_{i}^{*}(t)\right\}= & -a_{i i}(t)+b_{i i}(t) e^{-N_{i}^{*}(t)} \\
& +\sum_{j=1, j \neq i}^{n}\left(a_{i j}(t)-b_{i j}(t) e^{-N_{j}^{*}(t)}\right) \\
& +\sum_{j=1}^{l} c_{i j}(t) N_{i}^{*}\left(t-\tau_{i j}(t)\right) e^{-\gamma_{i j}(t) N_{i}^{*}\left(t-\tau_{i j}(t)\right)}, \quad i \in \Lambda .
\end{aligned}
$$

Therefore, $N^{*}(t)$ is a solution of (1.4).

Next we prove that $N^{*}(t)$ is an almost periodic solution of (1.4). From Lemma 2.2, for any $\varepsilon>0$, there exists $l=l(\varepsilon)>0$ such that every interval $[\alpha, \alpha+l]$ contains at least one number $\delta$ for which there exists $N>0$ satisfies

$$
\|v(t+\delta)-v(t)\| \leq \varepsilon \quad \text { for all } t>N
$$

Then, for any fixed $s \in R$, we can find a sufficiently large positive integer $N_{1}>N$ such that for any $k>N_{1}$,

$$
s+t_{k}>N, \quad\left\|v\left(s+t_{k}+\delta\right)-v\left(s+t_{k}\right)\right\| \leq \varepsilon .
$$

Let $k \rightarrow+\infty$, we obtain

$$
\left\|N^{*}(s+\delta)-N^{*}(s)\right\| \leq \varepsilon
$$

which implies that $N^{*}(t)$ is an almost periodic solution of system (1.4).

Finally, we prove that $N^{*}(t)$ is globally exponentially stable.

Let $N(t)=N\left(t ; t_{0}, \varphi\right)$ and $y(t)=N(t)-N^{*}(t)=\left(y_{1}(t), y_{2}(t), \ldots, y_{n}(t)\right)^{T}$, where $y_{i}(t)=$ $N_{i}(t)-N_{i}^{*}(t), t \in\left[t_{0}-r_{i},+\infty\right), i \in \Lambda$. Then

$$
\begin{aligned}
y_{i}^{\prime}(t)= & b_{i i}(t)\left[e^{-N_{i}(t)}-e^{-N_{i}^{*}(t)}\right] \\
& -\sum_{j=1, j \neq i}^{n} b_{i j}(t)\left[e^{-N_{j}(t)}-e^{-N_{j}^{*}(t)}\right] \\
& +\sum_{j=1}^{l} c_{i j}(t)\left[N_{i}\left(t-\tau_{i j}(t)\right) e^{-\gamma_{i j}(t) N_{i}\left(t-\tau_{i j}(t)\right)}-N_{i}^{*}\left(t-\tau_{i j}(t)\right) e^{-\gamma_{i j}(t) N_{i}^{*}\left(t-\tau_{i j}(t)\right)}\right] .
\end{aligned}
$$

It follows from Lemma 2.1 that there exists $t_{\varphi, N^{*}}>t_{0}$ such that

$$
\kappa \leq N_{i}(t), \quad N_{i}^{*}(t) \leq M \quad \text { for all } t \in\left[t_{\varphi, N^{*}}-r_{i},+\infty\right), i \in \Lambda
$$

We consider the Lyapunov functional

$$
V_{i}(t)=\left|y_{i}(t)\right| e^{\lambda t}, \quad i \in \Lambda
$$


For all $t>t_{\varphi, N^{*}}$ and $i \in \Lambda$, calculating the upper left derivative of $V_{i}(t)$ along with the solution $y_{i}(t)$ of (3.9), we have

$$
\begin{aligned}
D^{-}\left(V_{i}(t)\right) \leq & \lambda\left|y_{i}(t)\right| e^{\lambda t}+b_{i i}(t)\left[e^{-N_{i}(t)}-e^{-N_{i}^{*}(t)}\right] \operatorname{sgn}\left(N_{i}(t)-N_{i}^{*}(t)\right) e^{\lambda t} \\
& +\sum_{j=1, j \neq i}^{n} b_{i j}(t)\left|e^{-N_{j}(t)}-e^{-N_{j}^{*}(t)}\right| e^{\lambda t}+\sum_{j=1}^{l} c_{i j}(t) e^{\lambda t} \\
& \times\left|N_{i}\left(t-\tau_{i j}(t)\right) e^{-\gamma_{i j}(t) N_{i}\left(t-\tau_{i j}(t)\right)}-N_{i}^{*}\left(t-\tau_{i j}(t)\right) e^{-\gamma_{i j}(t) N_{i}^{*}\left(t-\tau_{i j}(t)\right)}\right| .
\end{aligned}
$$

In the sequel, we claim that

$$
\begin{aligned}
V_{i}(t) & =\left|y_{i}(t)\right| e^{\lambda t} \\
& <e^{\lambda t_{\varphi, N^{*}}}\left(\max _{i \in \Lambda} \max _{t \in\left[t_{0}-r_{i}, t_{\left.\varphi, N^{*}\right]}\right.}\left|N_{i}(t)-N_{i}^{*}(t)\right|+1\right) \\
& :=K_{\varphi, N^{*}} \quad \text { for all } t>t_{\varphi, N^{*}}, i \in \Lambda .
\end{aligned}
$$

Contrarily, there must exist $s_{7}>t_{\varphi, N^{*}}$ and $i \in \Lambda$ such that

$$
V_{i}\left(s_{7}\right)=K_{\varphi, N^{*}} \quad \text { and } \quad V_{j}(t)<K_{\varphi, N^{*}} \quad \text { for all } t \in\left[t_{0}-r_{j}, s_{7}\right), j \in \Lambda
$$

Since

$$
\kappa \leq \gamma_{i j}\left(s_{7}\right) N_{i}\left(s_{7}-\tau_{i j}\left(s_{7}\right)\right), \gamma_{i j}\left(s_{7}\right) N_{i}^{*}\left(s_{7}-\tau_{i j}\left(s_{7}\right)\right) \leq \gamma_{i j}^{+} M \leq \widetilde{\kappa}, \quad j=1,2, \ldots, l,
$$

together with (2.20)-(2.22), (3.10), (3.12) and (3.14), we get

$$
\begin{aligned}
0 \leq & D^{-}\left(V_{i}\left(s_{7}\right)\right) \\
\leq & \lambda\left|y_{i}\left(s_{7}\right)\right| e^{\lambda s_{7}}+b_{i i}\left(s_{7}\right)\left[e^{-N_{i}\left(s_{7}\right)}-e^{-N_{i}^{*}\left(s_{7}\right)}\right] \operatorname{sgn}\left(N_{i}\left(s_{7}\right)-N_{i}^{*}\left(s_{7}\right)\right) e^{\lambda s_{7}} \\
& +\sum_{j=1, j \neq i}^{n} b_{i j}\left(s_{7}\right)\left|e^{-N_{j}\left(s_{7}\right)}-e^{-N_{j}^{*}\left(s_{7}\right)}\right| e^{\lambda s_{7}}+\sum_{j=1}^{l} c_{i j}\left(s_{7}\right) e^{\lambda s_{7}} \\
& \times\left|N_{i}\left(s_{7}-\tau_{i j}\left(s_{7}\right)\right) e^{-\gamma_{i j}\left(s_{7}\right) N_{i}\left(s_{7}-\tau_{i j}\left(s_{7}\right)\right)}-N_{i}^{*}\left(s_{7}-\tau_{i j}\left(s_{7}\right)\right) e^{-\gamma_{i j}\left(s_{7}\right) N_{i}^{*}\left(s_{7}-\tau_{i j}\left(s_{7}\right)\right)}\right| \\
\leq & \lambda\left|y_{i}\left(s_{7}\right)\right| e^{\lambda s_{7}}-b_{i i}\left(s_{7}\right) e^{-M}\left|y_{i}\left(s_{7}\right)\right| e^{\lambda s_{7}}+\sum_{j=1, j \neq i}^{n} b_{i j}\left(s_{7}\right) e^{-\kappa}\left|y_{j}\left(s_{7}\right)\right| e^{\lambda s_{7}} \\
& +\sum_{j=1}^{l} c_{i j}\left(s_{7}\right) \mid N_{i}\left(s_{7}-\tau_{i j}\left(s_{7}\right)\right) e^{-\gamma_{i j}\left(s_{7}\right) N_{i}\left(s_{7}-\tau_{i j}\left(s_{7}\right)\right)} \\
& -N_{i}^{*}\left(s_{7}-\tau_{i j}\left(s_{7}\right)\right) e^{-\gamma_{i j}\left(s_{7}\right) N_{i}^{*}\left(s_{7}-\tau_{i j}\left(s_{7}\right)\right)} \mid e^{\lambda s_{7}} \\
= & -\left[b_{i i}\left(s_{7}\right) e^{-M}-\lambda\right] V_{i}\left(s_{7}\right)+\sum_{j=1, j \neq i}^{n} b_{i j}\left(s_{7}\right) e^{-\kappa} V_{j}\left(s_{7}\right) \\
& +\sum_{j=1}^{l} \frac{c_{i j}\left(s_{7}\right)}{\gamma_{i j}\left(s_{7}\right)} \mid \gamma_{i j}\left(s_{7}\right) N_{i}\left(s_{7}-\tau_{i j}\left(s_{7}\right)\right) e^{-\gamma_{i j}\left(s_{7}\right) N_{i}\left(s_{7}-\tau_{i j}\left(s_{7}\right)\right)} \\
& -\gamma_{i j}\left(s_{7}\right) N_{i}^{*}\left(s_{7}-\tau_{i j}\left(s_{7}\right)\right) e^{-\gamma_{i j}\left(s_{7}\right) N_{i}^{*}\left(s_{7}-\tau_{i j}\left(s_{7}\right)\right)} \mid e^{\lambda s_{7}}
\end{aligned}
$$




$$
\begin{aligned}
& \leq-\left[b_{i i}\left(s_{7}\right) e^{-M}-\lambda\right] V_{i}\left(s_{7}\right)+\sum_{j=1, j \neq i}^{n} b_{i j}\left(s_{7}\right) e^{-\kappa} V_{j}\left(s_{7}\right)+\sum_{j=1}^{l} c_{i j}\left(s_{7}\right) \frac{1}{e^{2}} V_{i}\left(s_{7}-\tau_{i j}\left(s_{7}\right)\right) e^{\lambda \tau_{i j}\left(s_{7}\right)} \\
& \leq\left\{-\left[b_{i i}\left(s_{7}\right) e^{-M}-\lambda\right]+\sum_{j=1, j \neq i}^{n} b_{i j}\left(s_{7}\right) e^{-\kappa}+\frac{1}{e^{2}} \sum_{j=1}^{l} c_{i j}\left(s_{7}\right) e^{\lambda r_{i}}\right\} K_{\varphi, N^{*} .}
\end{aligned}
$$

Thus,

$$
0 \leq-\left[b_{i i}\left(s_{7}\right) e^{-M}-\lambda\right]+\sum_{j=1, j \neq i}^{n} b_{i j}\left(s_{7}\right) e^{-\kappa}+\frac{1}{e^{2}} \sum_{j=1}^{l} c_{i j}\left(s_{7}\right) e^{\lambda r_{i}},
$$

which contradicts (2.14). Hence (3.13) holds. It follows that

$$
\left|y_{i}(t)\right|<K_{\varphi, N^{*}} e^{-\lambda t} \quad \text { for all } t>t_{\varphi, N^{*}}, i \in \Lambda
$$

This completes the proof.

\section{An example}

In this section, we give an example and numerical simulations to explain the results obtained in the previous sections.

Example 4.1 Consider the following Nicholson's blowflies system with nonlinear densitydependent mortality terms and patch structure:

$$
\left\{\begin{aligned}
N_{1}^{\prime}(t)= & -e^{-\left(1+0.08 \cos ^{2} \sqrt{2} t\right)}+(1+0.001 \cos \sqrt{3} t) e^{-N_{1}(t)}+0.1 e^{-\left(1+0.08 \sin ^{2} \sqrt{2} t\right)} \\
& -(0.1+0.0001 \sin \sqrt{3} t) e^{-N_{2}(t)}+\frac{1+\cos ^{2} t}{2,000} N_{1}\left(t-2 \sin ^{4} t\right) e^{-N_{1}\left(t-2 \sin ^{4} t\right)} \\
& +\frac{1+\cos ^{2} 2 t}{2,000} N_{1}\left(t-2 \cos ^{4} t\right) e^{-N_{1}\left(t-2 \cos ^{4} t\right)} \\
N_{2}^{\prime}(t)= & -e^{-\left(1+0.08 \sin ^{2} \sqrt{2} t\right)}+(1+0.001 \sin \sqrt{3} t) e^{-N_{2}(t)}+0.1 e^{-\left(1+0.08 \cos ^{2} \sqrt{2} t\right)} \\
& -(0.1+0.0001 \cos \sqrt{3} t) e^{-N_{1}(t)}+\frac{1+\sin ^{2} t}{2,000} N_{2}\left(t-2 \cos ^{4} t\right) e^{-N_{2}\left(t-2 \cos ^{4} t\right)} \\
& +\frac{1+\sin ^{2} 2 t}{2,000} N_{2}\left(t-2 \sin ^{4} t\right) e^{-N_{2}\left(t-2 \sin ^{4} t\right)}
\end{aligned}\right.
$$

Obviously, $r_{1}=r_{2}=2, a_{11}^{-}=a_{22}^{-}=e^{-1.08}, a_{11}^{+}=a_{22}^{+}=e^{-1}, a_{12}^{-}=a_{21}^{-}=0.1 e^{-1.08}, a_{12}^{+}=a_{21}^{+}=$ $0.1 e^{-1}, b_{11}^{-}=b_{22}^{-}=0.999, b_{22}^{+}=b_{22}^{+}=1.001, b_{12}^{-}=b_{21}^{-}=0.0999, b_{12}^{+}=b_{21}^{+}=0.1001, c_{11}^{-}=c_{12}^{-}=$ $c_{21}^{-}=c_{22}^{-}=0.0005, c_{11}^{+}=c_{12}^{+}=c_{21}^{+}=c_{22}^{+}=0.001, \gamma_{11}=\gamma_{12}=\gamma_{21}=\gamma_{22}=1$. Let $M=1.3$, from (2.1), we obtain $\widetilde{\kappa} \approx 1.342276, \kappa \approx 0.7215355$, and

$$
\begin{aligned}
& 1=\gamma_{i j}^{+} \leq \frac{\widetilde{\kappa}}{M} \approx \frac{1.342276}{1.3}, \quad i, j=1,2, \\
& \sup _{t \in R}\left\{-a_{i i}(t)+b_{i i}(t) e^{-M}+\sum_{j=1, j \neq i}^{2} a_{i j}(t)+\sum_{j=1}^{2} \frac{c_{i j}(t)}{\gamma_{i j}(t)} \frac{1}{e}\right\} \\
& \quad \leq-a_{i i}^{-}+b_{i i}^{+} e^{-M}+\sum_{j=1, j \neq i}^{2} a_{i j}^{+}+\sum_{j=1}^{2} \frac{c_{i j}^{+}}{\gamma_{i j}^{-}} \frac{1}{e} \\
& \approx-0.0293<0, \quad i=1,2,
\end{aligned}
$$




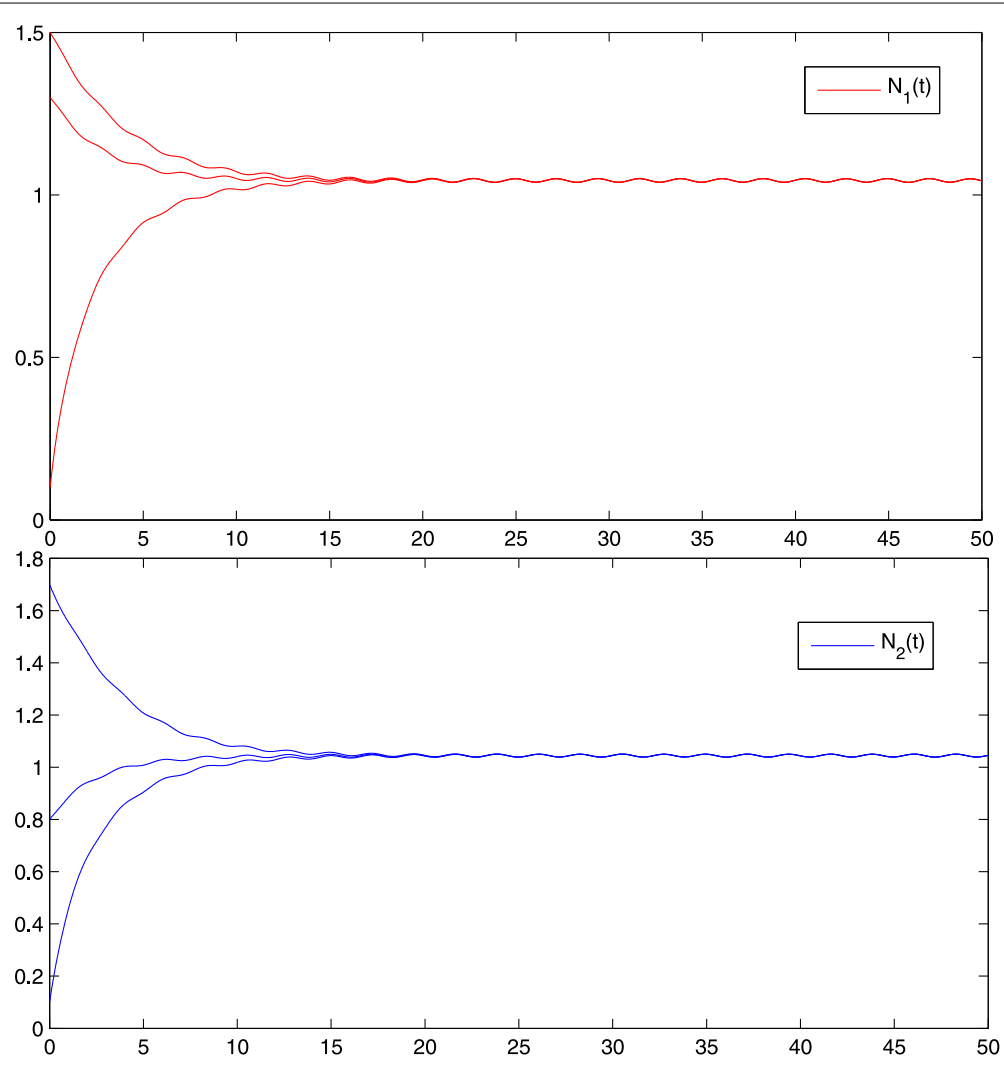

Figure 1 Numerical solutions $N(t)=\left(N_{1}(t), N_{2}(t)\right)^{T}$ of system (4.1) for the initial value $\varphi(t) \equiv(1.5,1.7)^{T},(1.3,0.8)^{T},(0.1,0.1)^{T}$.

$$
\begin{aligned}
& \inf _{t \in R, s \in[0, \kappa]}\left\{-a_{i i}(t)+b_{i i}(t) e^{-s}+\sum_{j=1, j \neq i}^{2}\left(a_{i j}(t)-b_{i j}(t)\right)+\sum_{j=1}^{2} \frac{c_{i j}(t)}{\gamma_{i j}(t)} s e^{-s}\right\} \\
& \geq-a_{i i}^{+}+b_{i i}^{-} e^{-\kappa}+\sum_{j=1, j \neq i}^{2}\left(a_{i j}^{-}-b_{i j}^{+}\right) \\
& \approx 0.0515>0, \quad i=1,2, \\
& \sup _{t \in R}\left\{-b_{i i}(t) e^{-M}+\sum_{j=1, j \neq i}^{n} b_{i j}(t) e^{-\kappa}+\frac{1}{e^{2}} \sum_{j=1}^{l} c_{i j}(t)\right\} \\
& \leq-b_{i i}^{-} e^{-M}+\sum_{j=1, j \neq i}^{n} b_{i j}^{+} e^{-\kappa}+\frac{1}{e^{2}} \sum_{j=1}^{2} c_{i j}^{+} \\
& \approx-0.2233<0, \quad i=1,2,
\end{aligned}
$$

which imply that the Nicholson's blowflies system (4.1) satisfies (2.2), (2.3) and (2.11). Therefore, system (4.1) has a positive almost periodic solution $N^{*}(t)$ which is globally exponentially stable with the exponential convergent rate $\lambda \approx 0.1$. The numerical simulations in Figure 1 strongly support the consequence. 
Remark 4.1 To the best of our knowledge, few authors have considered the problems of the global exponential stability of positive almost periodic solutions for a Nicholson's blowflies system with nonlinear density-dependent mortality terms and patch structure. It is clear that all the results in [25-35] and the references therein cannot be applicable to prove that all the solutions of (4.1) converge exponentially to the positive almost periodic solution. This implies that the results of this paper are essentially new.

Competing interests

The authors declare that they have no competing interests.

\section{Authors' contributions}

The authors declare that the study was realized in collaboration with the same responsibility. Both authors read and approved the final version.

\section{Author details}

'School of Mathematics and Information, Shanghai Lixin University of Commerce, Shanghai, 201620, People's Republic of China. ${ }^{2}$ College of Mathematics, Physics and Information Engineering, Jiaxing University, Jiaxing, Zhejiang 314001, People's Republic of China.

\section{Acknowledgements}

We would like to thank the anonymous reviewer for their suggestions to improve the manuscript. This work was supported by the National Natural Science Foundation of China (Grant No. 11301341, 11201184), Innovation Program of Shanghai Municipal Education Commission (Grant No. 13YZ127), and Shanghai Fiscal Special Fund (Grant No. A4-4902-13-12).

Received: 25 March 2014 Accepted: 4 July 2014 Published: 4 August 2014

\section{References}

1. Nicholson, AJ: An outline of the dynamics of animal populations. Aust. J. Zool. 2, 9-25 (1954)

2. Gurney, WSC, Blythe, SP, Nisbet, RM: Nicholson's blowflies revisited. Nature 287, 17-21 (1980)

3. So, J, Yu, JS: Global attractivity and uniform persistence in Nicholson's blowflies. Differ. Equ. Dyn. Syst. 2, 11-18 (1994)

4. Kulenović, MRS, Ladas, G, Sficas, Y: Global attractivity in Nicholson's blowflies. Appl. Anal. 43, 109-124 (1992)

5. Kulenović, MRS, Ladas, G: Linearized oscillations in population dynamics. Bull. Math. Biol. 49(5), 615-627 (1987)

6. Feng, Q, Yan, J: Global attractivity and oscillation in a kind of Nicholson's blowflies. J. Biomath. 17, 21-26 (2002)

7. Gyori, I, Ladas, G: Oscillation Theory of Delay Differential Equations with Applications. Clarendon Press, New York (1991)

8. Gyori, I, Trofimchuk, S: On the existence of rapidly oscillatory solutions in the Nicholson blowflies equation. Nonlinear Anal. 48, 1033-1042 (2002)

9. Giang, D, Lenbury, Y, Seidman, T: Delay effects of population growth. J. Math. Anal. Appl. 305, 631-643 (2005)

10. Kulenović, MRS, Ladas, G, Sficas, Y: Global attractivity in population dynamics. Comput. Math. Appl. 18, $925-928$ (1989)

11. Kulenović, MRS, Ladas, G: Linearized oscillations in population dynamics. Bull. Math. Biol. 49, 615-627 (1987)

12. Kuang, Y: Delay Differential Equations with Applications in Population Dynamics. Mathematics in Science and Engineering, vol. 191. Academic Press, Boston (1993)

13. Liz, E: Four theorems and one conjecture on the global asymptotic stability of delay differential equation. In: The First 60 Years of Nonlinear Analysis of Jean Mawhin, pp. 117-129. Word Scientific, River Edge (2004)

14. Wei, J, Li, M: Hopf bifurcation analysis in a delayed Nicholson blowflies equation. Nonlinear Anal. 60(7), 1351-1367 (2005)

15. Li, M, Yan, J: Oscillation and global attractivity of generalized Nicholson's blowfly model. In: Differential Equations and Computational Simulations (Chengdu, 1999), pp. 196-201. Word Scientific, River Edge (2000)

16. Saker, S, Agarwal, S: Oscillation and global attractivity in a periodic Nicholson's blowflies model. Math. Comput. Model. 35, 719-731 (2002)

17. Li, J: Global attractivity in Nicholson's blowflies. Appl. Math. J. Chin. Univ. Ser. B 11, 425-434 (1996)

18. Chen, Y: Periodic solutions of delayed periodic Nicholson's blowflies models. Can. Appl. Math. Q. 11, 23-28 (2003)

19. Braverman, E, Kinzebulatov, D: Nicholson's blowflies equation with a distributed delay. Can. Appl. Math. Q. 14 107-128 (2006)

20. Li, J, Du, C: Existence of positive periodic solutions for a generalized Nicholson blowflies model. J. Comput. Appl. Math. 221, 226-233 (2008)

21. Li, WT, Fan, YH: Existence and global attractivity of positive periodic solutions for the impulsive delay Nicholson's blowflies model. J. Comput. Appl. Math. 201, 55-68 (2007)

22. Berezansky, L, Braverman, E, Idels, L: Nicholson's blowflies differential equations revisited: main results and open problems. Appl. Math. Model. 34, 1405-1417 (2010)

23. Fink, AM: Almost Periodic Differential Equations. Lecture Notes in Mathematics, vol. 377. Springer, Berlin (1974)

24. He, CY: Almost Periodic Differential Equation. Higher Education Publishing House, Beijing (1992) (in Chinese)

25. Chen, W, Liu, B: Positive almost periodic solution for a class of Nicholson's blowflies model with multiple time-varying delays. J. Comput. Appl. Math. 235, 2090-2097 (2011)

26. Wang, W, Wang, L, Chen, W: Existence and exponential stability of positive almost periodic solution for Nicholson-type delay systems. Nonlinear Anal., Real World Appl. 12, 1938-1949 (2011) 
27. Long, F: Positive almost periodic solution for a class of Nicholson's blowflies model with a linear harvesting term. Nonlinear Anal., Real World Appl. 13, 686-693 (2012)

28. Wang, W: Positive periodic solutions of delayed Nicholson's blowflies models with a nonlinear density-dependent mortality term. Appl. Math. Model. 36, 4708-4713 (2012)

29. Hou, X, Duan, L, Huang, Z: Permanence and periodic solutions for a class of delay Nicholson's blowflies models. Appl. Math. Model. 2012, 1537-1544 (2012)

30. Liu, B, Gong, S: Permanence for Nicholson-type delay systems with nonlinear density-dependent mortality terms. Nonlinear Anal., Real World Appl. 12, 1931-1937 (2011)

31. Chen, W: Permanence for Nicholson-type delay systems with patch structure and nonlinear density-dependent mortality terms. Electron. J. Qual. Theory Differ. Equ. 2012, 73 (2012)

32. Chen, W, Wang, L: Positive periodic solutions of Nicholson-type delay systems with nonlinear density- dependent mortality terms. Abstr. Appl. Anal. 2012, Article ID 843178 (2012). doi:10.1155/2012/843178

33. Chen, Z: Periodic solutions for Nicholson-type delay system with nonlinear density-dependent mortality terms. Electron. J. Qual. Theory Differ. Equ. 2013, 1 (2013)

34. Liu, B: Almost periodic solutions for a delayed Nicholson's blowflies model with a nonlinear density-dependent mortality term. Adv. Differ. Equ. 2014, 72 (2014). doi:10.1186/1687-1847-2014-72

35. Xu, YL: Existence and global exponential stability of positive almost periodic solutions for a delayed Nicholson's blowflies model. J. Korean Math. Soc. 51, 473-493 (2014)

36. Wang, W: Exponential extinction of Nicholson's blowflies system with nonlinear density-dependent mortality terms Abstr. Appl. Anal. 2012, Article ID 302065 (2012). doi:10.1155/2012/302065

37. Hale, JK, Verduyn Lunel, SM: Introduction to Functional Differential Equations. Springer, New York (1993)

38. Smith, HL: An Introduction to Delay Differential Equations with Applications to the Life Sciences. Springer, New York (2011)

doi:10.1186/1687-1847-2014-205

Cite this article as: Chen and Wang: Almost periodic solutions for a delayed Nicholson's blowflies system with nonlinear density-dependent mortality terms and patch structure. Advances in Difference Equations 2014 2014:205.

\section{Submit your manuscript to a SpringerOpen ${ }^{\circ}$ journal and benefit from:}

- Convenient online submission

- Rigorous peer review

- Immediate publication on acceptance

- Open access: articles freely available online

- High visibility within the field

- Retaining the copyright to your article 